\title{
Observations of light elements in massive stars
}

\author{
A. Kaufer \\ European Southern Observatory, Alonso de Cordova 3107, \\ Casilla 19001, Santiago de Chile \\ email: akaufer@eso.org
}

\begin{abstract}
Observations of light elements in hot massive stars are limited to few transitions of boron in the satellite-ultraviolet; lithium and beryllium are not observable at all. But because of its high sensitivity to the effects of rotational mixing, boron abundance determinations in massive stars have excelled as the definite test for evolutionary models with rotation. In this paper the observational evidence for rotational mixing in massive stars is reviewed and alternative interpretations are discussed.
\end{abstract}

Keywords. Stars: abundances, massive, rotation

\section{Introduction}

Despite their small number (only three stars out of a thousand have a mass larger than $8 \mathrm{M}_{\odot}$ ) and their small mass fraction (only $14 \%$ of the mass of all stars is found in massive stars with $M>8 \mathrm{M}_{\odot}$ ), massive stars are important because they inject into the ISM large amounts of radiation, mass, and mechanical energy in shortest time $(3-30 \mathrm{Myr})$ (Meynet 2008). Massive stars hence drive the chemical and dynamical evolution of the galaxies through nucleosynthesis and (re-)distribution of elements. Consequently, accurate galaxy evolution models require a detailed understanding of the internal and core properties of massive stars.

The observation of surface abundances of non-radioactive elements is an efficient probe for studying stellar evolution and verifying internal nuclear processes. The consideration of rotation in stellar evolution models predicts significant effects on the evolutionary tracks and surface abundances of massive stars (cf. Heger \& Langer 2000 and Maeder \& Meynet 2000). In particular the light trace elements lithium, beryllium, and boron are strongly affected by effects of rotationally induced mixing. Hence their observations and detailed surface abundance analyses are critical to test effects of rotational mixing. In the following an overview of the available observations of light elements in massive stars is given and the methods and achievable accuracies of abundance determinations from the observations are described. Then the observed light-element abundance patterns and their interpretation in context of stellar evolution models with rotation are discussed.

\section{Observations}

Lithium has been crucial to test models of cool stars at all possible stages of evolution, from pre-main sequence to the post-AGB stage and is conveniently observed through the resonance doublet at $6706 \AA$. Beryllium is observable in cool stars through the Be II $\lambda 3130$ resonance doublet but of limited use because of the considerably more difficult access from the ground to this spectral region in the near-UV. Boron is the most difficult light element to observe since all transitions of B I, B II, and B III are below the atmospheric 
cut-off at $300 \mathrm{~nm}$ in the satellite ultraviolet. Unfortunately, boron is at the same time the only light element observable in hot massive stars. Boron can be observed either through the B II $\lambda 1362$ resonance line or the B III $\lambda 2066$ resonance doublet line.

After a 5-year forced hiatus, STIS onboard HST is today and the mid-term future the only observational resource to study boron in hot massive stars. In the past boron abundances have been determined using the B II $\lambda 1362$ line by Boesgaard \& Heacox (1978) using Copernicus observations of 16 'normal' A and B stars, by Venn, Lambert, \& Lemke(1996) using IUE observations of $6 \mathrm{~B}$ and $\mathrm{A}$ sub/giants and supergiants, and by Cunha et al. (1997) using GHRS data from HST of 4 B stars in Orion.

The B II $\lambda 1362$ line turns out to be rather problematic for accurate abundance determinations: the line is blended with Si III, Ni II, V II, Zn III, and Fe III and the line is sensitive to NLTE corrections as reported by Cunha et al. (1997) and Venn et al. (2002). For the temperature range of mid-B type stars and earlier, where B III is the dominant ion above a temperature of $18 \mathrm{kK}$, the B III $\lambda 2066$ doublet line appears better suited: the lines are not blended and NLTE effects are small compared to the B II line.

Proffitt et al. (1999) were the first to move to the B III $\lambda 2066$ line to determine boron abundances and ${ }^{11} \mathrm{~B} /{ }^{10} \mathrm{~B}$ isotopic ratios for $3 \mathrm{~B}$ stars using very high-SNR GHRS spectra. Proffitt et al. (1999) find isotopic ratios of the $3 \mathrm{~B}$ stars of ${ }^{11} \mathrm{~B} /{ }^{10} \mathrm{~B} \sim 4$ consistent with the solar-system value (Anders \& Grevesse 1989). Proffitt \& Quigley (2001) explored the $I U E$ archive for high-resolution spectra of B-type stars and determined boron abundances (and upper limits) for 45 early B-type stars. Venn et al. (2002) determine 4 boron upper limits from 7 B-type main sequence stars using STIS onboard HST. Mendel et al. (2006) observe 7 additional B-type main sequence stars with STIS and find most of them boron depleted. Brooks et al. (2002) add to this handful of boron abundances of hot massive galactic B-type stars upper limits for boron abundances for two B-type stars in the Small Magellanic Cloud (SMC) obtained with STIS and the $H S T(V \approx 15 \mathrm{mag})$.

\section{Abundance analysis of hot stars}

To convert an observation of a stellar spectrum into an abundance measurement requires to carry out a detailed abundance analysis using atmosphere models and lineformation codes. For the early- to mid-B type main sequence stars, which are primarily observed in the context of light element abundances, the stellar atmospheres can be assumed to be plane-parallel and non-expanding, i.e., stellar winds are considered to be negligible. Then the stellar atmospheres can be described by 1D line-blanketed LTE models. Information on the gravity is obtained from the shape of the Balmer-line wings, which are broadened by the gravity-sensitive Stark effect. The line wings are fit with 1D (N)LTE Balmer line formation models. Effective temperature is derived from the so-called ionisation equilibrium of different ions of the same element (like Si II/III/IV) to reproduce the observed line profiles and equivalent widths for a given element abundance. Depending of the individual lines used for the analysis this requires 1D LTE line formation plus NLTE corrections or - if available - 1D NLTE line formation computations. Despite of sophisticated NLTE line-formation codes, for early-type B stars, usually a slight dependency of the computed line-by-line abundance on the strength of the lines remains, which is attributed to an additional line-broadening, the so-called microturbulence. It is determined from lines of ions that span a large range in equivalent widths, i.e., $\mathrm{O}$ or Fe in early B-type stars. Once effective temperature, gravity, and microturbulence have been determined in a quasi self-consistent way, the line-by-line element abundances can be determined using again 1D (N)LTE line formation. 
Nieva \& Przybilla (2007) and Nieva \& Przybilla (2008) have pushed this hybrid NLTE approach for detailed abundance analyses of early B-type stars to a high level of perfection. Przybilla, Nieva, \& Butler (2008) demonstrate that for a carefully selected sample of early-type B stars in the solar neighborhood with high-quality spectral observations, individual element abundances with 1 sigma errors clearly less than 0.1 dex can be obtained for all observable ions in the optical spectra of these stars.

A direct comparison of the proto-solar abundances of Asplund et al. (2009) with the B-star abundances of Przybilla, Nieva, \& Butler (2008) shows nearly perfect agreement:

$$
\begin{array}{llllll}
\text { B stars } & \mathrm{X}=0.715 & \mathrm{Y}=0.271 & \mathrm{Z}=0.014 & \mathrm{Z} / \mathrm{X}=0.020 & \text { (Przybilla et al. 2008) } \\
\text { proto-solar } & \mathrm{X}=0.7154 & \mathrm{Y}=0.2703 & \mathrm{Z}=0.0142 & \mathrm{Z} / \mathrm{X}=0.0198 \quad \text { (Asplund et al. 2009) }
\end{array}
$$

Considering these advances and the improved accuracies that can be obtained with such state-of-the-art abundance determination techniques, it seems advisable to make the effort to reanalyse all available (but still few) spectra of early-type B stars for which satellite ultraviolet observations of light elements, i.e., of boron are available.

\section{Boron abundances patterns in hot stars}

Most of the boron abundance studies of hot massive stars aim to establish the presentday boron abundance to constrain the Galactic chemical evolution models. Cunha (2010) reviews the implications of these observations of cool and hot stars for chemical evolution models and constraints on the boron production mechanisms.

The latter is of particular interest since boron is the only light element whose production is neither due to nucleosynthesis in stellar interiors nor due to big-bang nucleosynthesis but is produced through cosmic ray spallation reactions in the interstellar gas. Boron abundance trends as function of oxygen provide constraints on the possible spallation reactions at work.

Cunha et al. (2000) and Smith, Cunha, \& King (2001) find a linear trend for the boron abundances as function of oxygen abundances for galactic FG disk stars that show no beryllium depletion. The measured slope of $1.50 \pm 0.08$ (Cunha 2010) indicates a combination of primary source (expected slope 1) and secondary source (expected slope 2) production of boron in the Galaxy.

If the boron - oxygen abundances patterns determined for galactic hot stars by Proffitt \& Quigley (2001) and Mendel et al. (2006) are compared with the galactic FG disk stars, a good agreement is found for the non-boron depleted hot stars.

Interestingly, an extrapolation of the boron - oxygen trend with a slope of 1.5 to lowmetallicity environments is in excellent agreement with the abundance determinations (upper limits) of two B stars in the SMC by Brooks et al. (2002) (cf. their Fig. 6). The fact that boron in hot stars is frequently found to be severely depleted however complicates the rigorous interpretation of this interesting result. An increase in the sample size in the MCs would be the obvious demand to tighten the constraints on boron production by spallation in the MCs in comparison with the Galaxy but at the same time appears almost prohibitive considering the excessive amount of required STIS observing time (some 15 hours of HST integration time per star to obtain a $\mathrm{S} / \mathrm{N} \sim 50$ ).

Additional constraints on cosmic ray spallation theory could be obtained through high resolution, high SNR observations of the BIII $\lambda 2066$ line, which allows to determine ${ }^{11} \mathrm{~B} /{ }^{10} \mathrm{~B}$ isotopic ratios from the predicted isotopic shift of $42 \mathrm{~m} \AA$. The solar-system value of ${ }^{11} \mathrm{~B} /{ }^{10} \mathrm{~B} \sim 4$ (Anders \& Grevesse 1989) is larger than the prediction from current cosmic ray spallation theory $\left({ }^{11} \mathrm{~B} /{ }^{10} \mathrm{~B} \sim 2.5\right.$, Meneguzzi, Audouze, \& Reeves 1971). An observational determination of the isotopic ratio from hot massive stars would therefore 
provide strong constraints on the ${ }^{11} \mathrm{~B}$ production processes. Again, such observations are possible with STIS but demanding in observing time.

\section{Boron depletion in hot stars}

The light elements lithium, beryllium, and boron are very sensitive to destruction by proton capture at temperatures much lower than those where the $\mathrm{H}$-burning $\mathrm{CN}$-cycle is effective. This makes the light elements a sensitive tracer of mixing of stellar surface layers to deeper layers and can be observed as apparent depletion of the respective element. Lithium and beryllium have been intensively used in this context to study stellar evolution processes in cool stars but cannot be observed in hot stars. For hotter stars, boron is the only observable light element as discussed before.

Boron is a very fragile element and destroyed by proton capture already at temperatures of $<6 \times 10^{6} \mathrm{~K}$, which corresponds to about $1 \mathrm{M}_{\odot}$ below the surface of a B-type main-sequence star. Due to this sensitivity of boron to moderate temperatures, any mild mixing of surface layers to deeper layers would already deplete boron while keeping less fragile elements like nitrogen unchanged. In more evolved stages of the star the same mixing process may enhance other elements like nitrogen (progressive mixing). Therefore, the determination of boron - nitrogen abundance patterns in hot stars of different evolutionary stage is a sensitive method to test stellar evolution models.

Venn, Lambert, \& Lemke (1996) were the first to explore this possibility in a dedicated study of a sample of A and B stars of different evolutionary stage. Contrary to the expectation to find boron depletion for the more evolved stars showing at the same time nitrogen enrichment, the authors found severe boron depletion for all stars of their sample including for the non-evolved stars near the main sequence that show only little nitrogen enrichment. The finding of boron depletion without nitrogen enhancement could only be explained by invoking an additional mixing process. Based on their evolutionary tracks of massive stars including the effects of rotation, Fliegner, Langer, \& Venn (1996) proposed rotationally induced mixing acting in the radiative zones of massive main star-sequence stars to explain the observations of Venn, Lambert, \& Lemke (1996).

Figure 1 compiles all to date available boron and nitrogen abundance data of B-type stars from Venn et al. (2002), Lemke, Cunha, \& Lambert (2000), Proffitt et al. (1999), and Proffitt \& Quigley (2001). The dashed (blue) line shows an evolutionary track according to Heger \& Langer (2000) for a fast rotating massive star of $12 \mathrm{M}_{\odot}$ and $v_{\mathrm{eq}}=200 \mathrm{~km} / \mathrm{s}$, which nicely demonstrate the rapid depletion of boron by a factor of $\sim 50$ while nitrogen enhances by a factor of $\sim 2.5$ only. The newer boron observations have added three measurement of the two stars HD 36591 and HD 30836 to the group of stars showing strong boron depletion with virtually no nitrogen enrichment (group II in the classification of Morel, Hubrig, \& Briquet (2008)). These observations still remain difficult to explain by rotational mixing. However, new models of the Geneva stellar evolution code with rotational mixing and an extended reaction network including lithium, beryllium, and boron by Frischknecht (2010) promise to obtain an even faster boron depletion and therefore a better match to the observations.

It should be mentioned here that also other effects than rotational mixing can alter the stellar surface composition of hot massive stars: mass loss and mass transfer in binary systems are the primary candidates. While mass loss does not play a strong role in Btype main-sequence stars, the mass transfer in a close binary could produce considerable effects to the measured abundance patterns. For a detailed discussion of the latest results on element depletion and enhancements through binary interaction in massive stars see Langer (2010). 


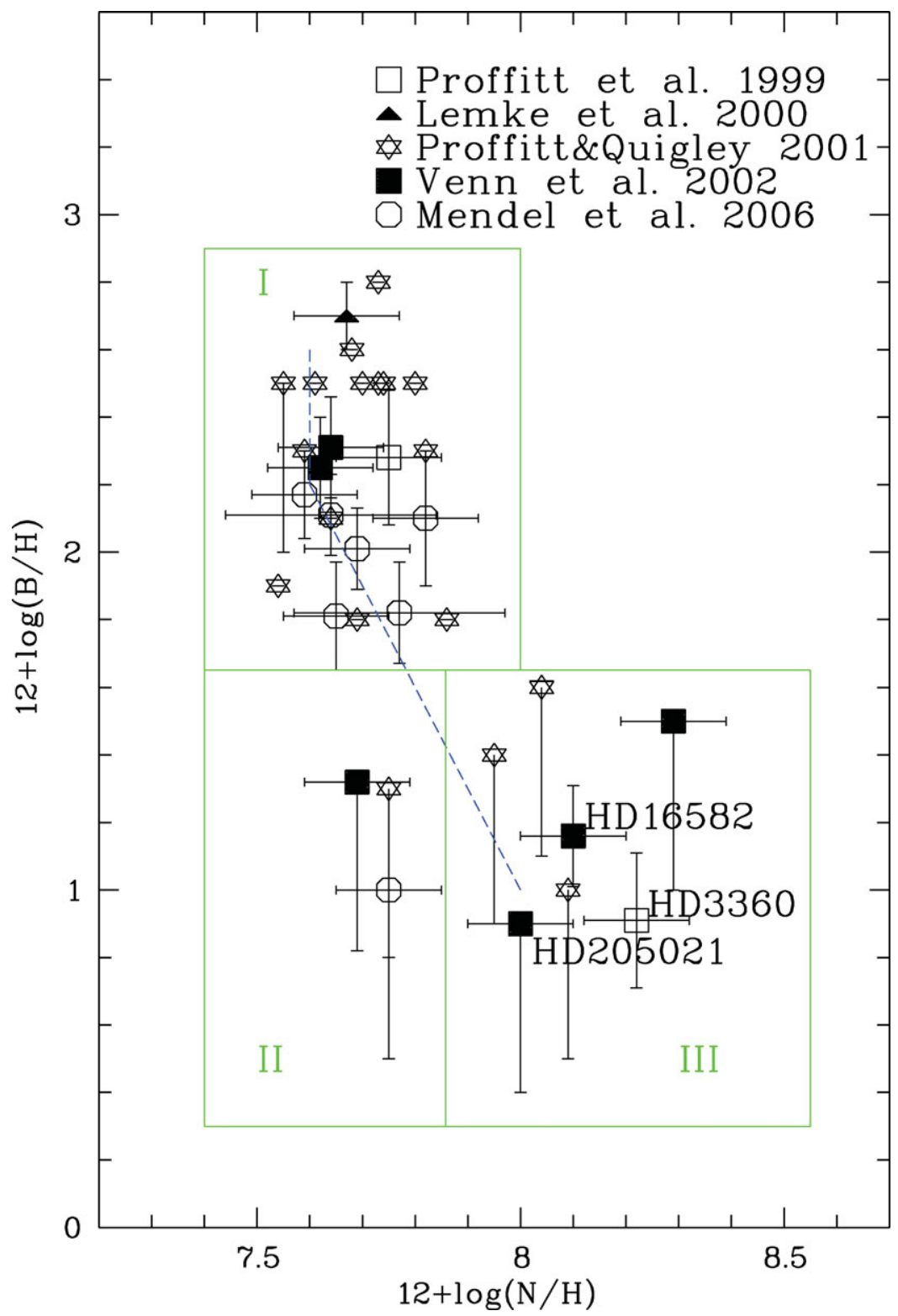

Figure 1. Boron and nitrogen abundances in B stars. Error bars of $+0 /-0.5$ dex indicate upper limits. The dashed (blue) line shows an evolutionary track according to Heger \& Langer (2000) for a rotating star of $12 \mathrm{M}_{\odot}$ and $v_{\mathrm{eq}}=200 \mathrm{~km} / \mathrm{s}$ and an initial nitrogen and boron abundance of 7.6 and 2.6, respectively. The (green) boxes indicate the different groups described in Morel, Hubrig, \& Briquet (2008), i.e., I - unaltered, close to solar nitrogen and boron abundances, II boron depleted but nitrogen unchanged, and III - boron depleted and nitrogen enhanced. Three stars are indicated with their HD number for which independent observations have established that these stars are slow rotators. See text for discussion. 
Despite the compelling results from boron - nitrogen abundance measurements and the striking evidence for rotational mixing in massive stars, further observations and precise abundance analyses would be highly desirable to verify that rotational mixing increases with age, rotation rates, and stellar mass. The revival of STIS during the spectacular service mission to HST in 2009 opens up new possibilities for such observations on larger samples of hot massive stars.

\section{Boron abundances and rotational velocities}

Observational tests of rotational mixing suffer from a fundamental dilemma: while measurable effects of altered abundance pattern are only predicted for fast rotating stars with $v_{\text {eq }} / v_{\text {crit }}>0.2$, detailed abundance analyses require sharp-lined stellar spectra. Therefore, abundance analyses are strongly biased towards sharp-line stars with $v_{\text {eq }} \sin i<20 \mathrm{~km} / \mathrm{s}$, i.e., stars either seen pole-on or real slow rotators. Considering that the median equatorial rotational velocity of B-type main-sequence stars is $\sim 200 \mathrm{~km} / \mathrm{s}$ (Howarth 2004), slow rotators cannot be considered representative of their class.

It is therefore crucial to include true fast rotators in future boron abundance studies by expanding the samples with stars showing rotationally broadened lines. Precise abundance analyses can still be carried out from spectra with rotationally broadened lines up to $v_{\text {eq }} \sin i \sim 100 \mathrm{~km} / \mathrm{s}$ if the SNR of the spectra is increased accordingly (cf. e.g. Kaufer et al. 1994 and references therein).

It is further crucial to determine true stellar rotation rates for the stars under examination to allow direct comparison with evolutionary models of rotating stars. The rotation of stellar surface patterns like stellar spots or non-radial pulsation patterns or the rotation of wind patterns locked to the stellar surface (cf. e.g. Prinja, Massa, \& Fullerton (1995), Fullerton et al. (1997), Kaufer et al. (2006)) can provide direct measurements of true stellar rotation rates. Rotational modulation can be more or less easily observed through spectral or photometrical time series studies of the respective bright galactic early-type B stars.

Morel, Hubrig, \& Briquet (2008) have applied this idea to the sample of early-type B stars for which boron and nitrogen abundances have been determined and which are shown in Fig. 1. The authors identify three boron depleted and nitrogen enriched stars (their group III) which are true slow rotators as determined from the modulation of UV wind lines (UV) or asteroseismological (AS) determination of the rotation rate:

$$
\begin{array}{lllll}
\text { HD } 3360 & \zeta \text { Cas } & v_{\text {eq }}=55 \mathrm{~km} / \mathrm{s} & \text { UV } & \text { SPB }+ \text { magn.field Neiner } \text { et al. }(2003) \\
\text { HD } 16582 & \delta \text { Cet } & v_{\text {eq }}=14 \text { or } 28 \mathrm{~km} / \mathrm{s} & \text { AS } & \beta \text { Cep }+ \text { magn.field Aerts } \text { et al. }(2006) \\
\text { HD 205021 } & \beta \text { Cep } & v_{\text {eq }}=26 \mathrm{~km} / \mathrm{s} & \text { UV } & \beta \text { Cep }+ \text { magn.field Henrichs } \text { et al. }(2000)
\end{array}
$$

The fact that all three stars are either slowly pulsating B stars or $\beta$ Cephei stars for which the presence of a weak magnetic field has been established leads Morel, Hubrig, \& Briquet (2008) to propose that magnetic phenomena are important in altering the photospheric abundances of early B dwarfs, even for surface field strengths as low as at the one hundred Gauss level. The authors further argue that these stars only suffered a moderate amount of angular momentum loss along the main sequence and that they were likely slow rotators already on the ZAMS. At least in the cases of these three stars, the boron depletion can therefore not be explained by rotational mixing, which otherwise nicely reproduces the transition from group I to group III as illustrated in Fig. 1 by the evolutionary track from Heger \& Langer (2000). 


\section{Conclusions}

Undoubtedly the observational discovery of an additional mixing process to explain the boron depletion in the surface layers of non-evolved hot B stars on or near the main sequence by Venn, Lambert, \& Lemke (1996) has been the most notable contribution by the studies of the light elements in massive stars. The subsequent identification of this mixing process to be due to rotationally induced mixing in the radiative layers of massive stars by Fliegner, Langer, \& Venn (1996) has to be considered a major milestone in the development of stellar evolution models of massive stars. The understanding of the importance of stellar rotation for the evolution of massive stars has revolutionised the whole field of hot star research. Still, the number of actual measurements of boron in massive stars remains scarce due to the observational limitation imposed by the diagnostic lines in the satellite ultraviolet. The recent return of STIS onboard HST into science operation presents a rather unique opportunity to intensify the studies of rotational mixing as function of age, mass, and rotational rates of massive stars. Large and carefully selected samples of early-type stars should be examined to establish statistically significant dependencies on the relevant evolution parameters. The Galactic and MC samples already studied by the VLT-FLAMES survey of massive stars (Hunter el al. 2009) appear to be best suited for such future studies since they cover a large range of rotational velocities up to $300 \mathrm{~km} / \mathrm{s}$ and a wide range of ages and masses.

\section{References}

Aerts, C. et al. 2006, ApJ, 642, 470

Anders, E. \& Grevesse, N. 1989, GeCoA, 53, 197

Asplund, M., Grevesse, N., Sauval, A. J., \& Scott, P. 2009, ARA\& A, 47, 481

Boesgaard, A. M. \& Heacox, W. D. 1978, ApJ, 226, 888

Brooks, A. M., Venn, K. A., Lambert, D. L., Lemke, M., Cunha, K., \& Smith, V. V. 2002, ApJ, 573,584

Cunha, K., Lambert, D. L., Lemke, M., Gies, D. R., \& Roberts, L. C. 1997, ApJ, 478, 211

Cunha, K., Smith, V. V., Parizot, E., \& Lambert, D. L. 2000, ApJ, 543, 850

Cunha, K. 2010, these proceedings

Fliegner, J., Langer, N., \& Venn, K. A. 1996, A\&AA, 308, L13

Frischknecht, U. 2010, these proceedings

Fullerton, A. W., Massa, D. L., Prinja, R. K., Owocki, S. P., \& Cranmer, S. R. 1997, A $\varepsilon A, 327$, 699

Heger, A. \& Langer, N. 2000, ApJ, 544, 1016

Henrichs, H. F. et al. 2000, ASPC, 214, 324

Howarth, I. D. 2004, IAUS, 215, 33

Hunter, I. et al. 2009, A\&A, 496, 841

Kaufer, A., Szeifert, T., Krenzin, R., Baschek, B., \& Wolf, B. 1994, A\&SA, 289, 740

Kaufer, A., Stahl, O., Prinja, R. K., \& Witherick, D. 2006, A\& A, 447, 325

Langer, N. 2010, these proceedings

Lemke, M., Cunha, K., \& Lambert, D. L. 2000, LIACo, 35, 223

Maeder, A. \& Meynet, G. 2000, A\&A A, 361, 159

Mendel, J. T., Venn, K. A., Proffitt, C. R., Brooks, A. M., \& Lambert, D. L. 2006, ApJ, 640, 1039

Meneguzzi, M., Audouze, J., \& Reeves, H. 1971, A\&A, 15, 337

Meynet, G. 2008, EAS Publication Series, 32, 187

Morel, T., Hubrig, S., \& Briquet, M. 2008, A\&A, 481, 453

Neiner, C., Geers, V. C., Henrichs, H. F., Floquet, M., Frémat, Y., Hubert, A.-M., Preuss, O., \& Wiersema, K. 2003, A\& A, 406, 1019

Nieva, M. F. \& Przybilla, N. 2008, A\&A, 481, 199 
Nieva, M. F. \& Przybilla, N. 2007, A\&A, 467, 295

Prinja, R. K., Massa, D., \& Fullerton, A. W. 1995, ApJ, 452, L61

Proffitt, C. R., Jönsson, P., Litzén, U., Pickering, J. C., \& Wahlgren, G. M. 1999, ApJ, 516, 342

Proffitt, C. R. \& Quigley, M. F. 2001, ApJ, 548, 429

Przybilla, N., Nieva, M.-F., \& Butler, K. 2008, ApJ, 688, L103

Smith, V. V., Cunha, K., \& King, J. R. 2001, AJ, 122, 370

Venn, K. A., Lambert, D. L., \& Lemke, M. 1996, A\&A, 307, 849

Venn, K. A., Brooks, A. M., Lambert, D. L., Lemke, M., Langer, N., Lennon, D. J., \& Keenan, F. P. 2002, ApJ, 565, 571 